TITLE:

\title{
Reexamination of the equilibrium melting temperature of natural rubber crystals
}

\author{
$\operatorname{AUTHOR}(S)$ :
}

Tosaka, Masatoshi; Kumagawa, Daiki

\section{CITATION:}

Tosaka, Masatoshi ...[et al]. Reexamination of the equilibrium melting temperature of natural rubber crystals. Polymer Journal 2020, 52: 255259

ISSUE DATE:

2020-02

URL:

http://hdl.handle.net/2433/245628

\section{RIGHT:}

This is the accepted manuscript of the article, which has been published in final form at https://doi.org/10.1038/s41428019-0253-9.; The full-text file will be made open to the public on 1 August 2020 in accordance with publisher's 'Terms and Conditions for Self-Archiving'.; This is not the published version. Please cite only the published version.; この論文は 出版社版でありません。引用の際には出版社版をご確認ご利用ください。 


\section{Reexamination of the equilibrium melting temperature of natural \\ rubber crystals}

\section{Running head}

Equilibrium melting temperature of natural rubber

\section{Key words}

Small angle X-ray scattering, Correlation function, Deproteinization, Seeding, Nucleation

\section{Authors}

Masatoshi Tosaka*, Daiki Kumagawa

\section{Affiliation}

Institute for Chemical Research, Kyoto University, Gokasho, Uji, Kyoto-fu 611-0011, Japan

* Corresponding author

Tel: +81-774-38-3062

Fax: +81-774-38-3067

E-mail: tosaka@scl.kyoto-u.ac.jp 


\section{Abstract}

Equilibrium melting temperature $\left(T_{m}^{0}\right)$ of natural rubber crystal was reexamined by the Hoffman-Weeks plot and the Gibbs-Thomson plot using lamellar thickness obtained from the same sample as the one used for the measurement of melting temperature. The sample was prepared from deproteinized latex. As a result, $T_{m}^{0}$ of NR was estimated to be $61{ }^{\circ} \mathrm{C}$. At the same time, fold-surface free energy was estimated to be $0.046 \mathrm{Jm}^{-2}$. These values should be more reliable than the previously assumed ones.

\section{Introduction}

Natural rubber (NR) is the most widely-used elastomer due to its favorable features such as large elongation at break, tensile strength, along with resistance against crack growth and wear. These good mechanical properties are thought to be related to strain-induced crystallization of NR. There have been numerous studies that try to describe strain-induced crystallization on the basis of thermodynamics of polymer crystals, though none of them is successful in terms of versatility to various samples. For this kind of attempt, equilibrium melting temperature $\left(T_{m}^{0}\right)$ is one of important parameters that describe the crystallization and melting behaviors.

It is well known that crystalline linear polymer usually forms lamellar crystals in which polymer chains are aligned normal to the basal plane and fold back and forth on the upper and lower fold surfaces. Due to the high energy for the formation of the fold surfaces, melting temperature $\left(T_{m}\right)$ of lamellar polymer crystals are depressed from $T_{m}^{0}$. In other words, $T_{m}^{0}$ reflects the inherent melting property of polymer crystals.

Roberts and Mandelkern [1] reported "equilibrium melting temperature” of NR to be ca. 28 ${ }^{\circ}$ C. (Strictly speaking, the term "cis-1,4-polyisoprene” should be used instead of NR [2]. For simplicity, however, we use the term NR.) Because this study was published (in 1955) before the recognition of lamellar morphology of polymer crystals by the study of Keller [3] (1957), 
the meaning and estimation method of "equilibrium melting temperature” were different from currently accepted ones. Krigbaum et al. [4] estimated $T_{m}^{0}$ of NR to be ca. $50{ }^{\circ} \mathrm{C}$. In this work, the $T_{m}^{0}$ value for the unstrained NR was estimated by extrapolation of the values for stretched samples. The above-mentioned values have been rarely cited in literatures. Estimation of $T_{m}^{0}$ of NR in quiescent state has been performed by Dalal et al. [5] and Kawahara, et al. [6] on the basis of the Gibbs-Thomson equation [7],

$$
T_{m}=T_{m}^{0}\left(1-\frac{2 \sigma_{e}}{l \Delta H}\right)
$$

where $\sigma_{e}$ is the fold-surface energy per area, $\Delta H$ is the melting enthalpy change and $l$ is the thickness of lamellar crystals. $T_{m}^{0}$ can be estimated by extrapolating the plot of $T_{m}$ versus $1 / l$ to $1 / l=0$, namely, to infinite lamellar thickness. The value of $T_{m}^{0}$ was estimated to be $35-36{ }^{\circ} \mathrm{C}$ in these studies, and this $T_{m}^{0}$ value may have beem most cited (for example, in refs. [8-12]). It is noted that Dalal et al. [5] and Kawahara, et al. [6] did not obtained the values of $l$ from the bulk samples used for the melting experiments; the values of $l$ were calculated using an empirical equation which relates crystallization temperature $\left(T_{c}\right)$ with $l$ values of lamellar crystals in thin-film specimens, which were measured by transmission electron microscopy.[5]

By definition, $T_{m}^{0}$ is the highest attainable $T_{m}$, and accordingly, melting of a crystal above $T_{m}^{0}$ should not occur. However, there are some articles reporting melting of NR crystals at temperature higher than $36{ }^{\circ} \mathrm{C}$, namely, above the currently-used $T_{m}^{0}$ value. For example, Valladares et al. [11] observed melting of spherulites in unstrained NR from 39 to $45{ }^{\circ} \mathrm{C}$. Katzenberg et al. [13] and Tosaka et al. [14] also observed melting above $40{ }^{\circ} \mathrm{C}$ for straininduced crystals of NR. These observations suggest that the real value of $T_{m}^{0}$ must be higher than $45{ }^{\circ} \mathrm{C}$. The discrepancy may have occurred because the values of $l$ and $T_{m}$ were obtained from different specimen. Therefore, in this study, we reexamined $T_{m}^{0}$ of NR avoiding this problem. 


\section{Experimental}

\section{Materials}

NR latex (dry rubber content 60\%) was purchased from Sime Darby Research Sdn. Bhd., Malaysia. The latex was deproteinized using urea by the following procedure [15]. To the weight of NR latex, $0.1 \mathrm{wt} \%$ of urea and $1 \mathrm{wt} \%$ of sodium dodecyl sulfate (SDS) were mixed. After incubation for $1 \mathrm{~h}$ at room temperature, the latex was centrifuged at 10,000G for $1 \mathrm{~h}$. The upper cream fraction was redispersed in $1 \mathrm{wt} \%$ aqueous solution of SDS to make $30 \%$ latex and was washed twice by centrifugation to prepare deproteinized NR. Before and after the treatment, the NR sample was examined by FT-IR spectroscopy. The deproteinized latex was poured into a flat mold and dried to form a uniform NR sheet (thickness $1 \mathrm{~mm}$ ). The NR sheets were cut into small pieces for the subsequent measurements and stored in a refrigerator kept at ca. $10{ }^{\circ} \mathrm{C}$ for a month or longer in order to grow seed crystals of NR.

In our preliminary examination, fatty acids in the deproteinized NR were also removed by Soxhlet extraction with acetone. However, the latter samples did not crystallize after one month of storage in the refrigerator, and accordingly, they were not used in the current study.

\section{FT-IR spectroscopy}

FT-IR spectroscopy measurements were performed using a Shimadzu IRPrestige-21. Thin films of untreated or deproteinized NR latex was used as the specimen.

\section{Differential scanning calorimetry for Hoffman-Weeks plot}

A series of differential scanning calorimetry (DSC) measurements was performed using a conventional instrument (DSC-60, Shimadzu Co.) in nitrogen gas atmosphere. In order to form NR crystals at predetermined crystallization temperature $\left(T_{c}\right)$ in reasonable range of time, seed 
crystals were used that reduce induction period of nucleation. About $10 \mathrm{mg}$ of the NR sample which has been crystallized in the refrigerator was crimped in an aluminum pan and used for the measurements. A care was taken in order not to completely melt the seed crystals in the NR sample. By a preliminary scan, $T_{m}$ of the seed crystals was confirmed. After that, the temperature program of the DSC measurements was set as in Fig. 1a. First, the sample was heated up to temperature slightly below complete melting of the seed crystals (see Fig. 1b). Next, the sample was cooled to $T_{c}$ (between $-37{ }^{\circ} \mathrm{C}$ and $7{ }^{\circ} \mathrm{C}$ ) at the rate of $10{ }^{\circ} \mathrm{C} / \mathrm{min}$ and held for more than 60 minutes (up to $180 \mathrm{~min}$ ) to form crystals at this temperature. Then the sample was cooled down to $-50{ }^{\circ} \mathrm{C}$ and the second heating scan at the rate of $10{ }^{\circ} \mathrm{C} / \mathrm{min}$ was started. An example of the DSC scans is shown in Fig. 1b. Two melting peaks were observed during the second heating scan. The one appeared at the lower temperature corresponds to the crystals formed at $T_{c}$, and the other at the higher temperature corresponds to the seed crystal.

\section{Combined DSC/X-ray measurement for Gibbs-Thomson plot}

For small angle X-ray scattering (SAXS) measurements, a conventional instrument (NanoViewer, operated at $40 \mathrm{kV}, 30 \mathrm{~mA}$, Rigaku Co.) equipped with a semiconductor detector (PILATUS 100K, Dectris) was used. The wavelength $(\lambda)$ of the incident X-ray was $0.154 \mathrm{~nm}$ and the camera length was about $850 \mathrm{~mm}$. The sample was crimped in a special aluminum pan that has a hole in the center for X-ray measurement; the size of the sample was larger than the hole. Then the sample was set in a XRD-DSC attachment (Thermo plus EVO II Series XRDDSC Attachment for Transmission Measurement, Rigaku Co.). The temperature program for the DSC measurement was almost the same as the case of the independent DSC measurements using the conventional instrument. In this case, however, SAXS measurement was additionally performed just after crystallization at $T_{c}$ (between $-35{ }^{\circ} \mathrm{C}$ and $-4{ }^{\circ} \mathrm{C}$ ). Therefore, the length of time before the second DSC heating scan (namely, crystallization time) was by about 30 min 
longer than the case using the conventional DSC instrument. Additionally, cooling rate of the XRD-DSC attachment was much slower (of the order of $1{ }^{\circ} \mathrm{C} / \mathrm{min}$ ), and accordingly, the longer time was required before attaining $T_{c}$.

The two-dimensional SAXS patterns were converted into one-dimensional intensity profile by 2DP software (Rigaku Co.). Fourier transformation in order to convert the intensity profile to the electron density correlation function [16] was performed by our self-made software.

\section{Results and discussion}

It is well known that the plant-derived NR contains non-rubber components such as proteins (1-2 \% in wt/vol of latex) and fatty acids (0.9-1.7\%) [2]. Removal of proteins from the latex was confirmed by the FT-IR measurement. As shown in Fig. 2, specific protein bands (indicated by arrows in Fig. 2) measured at $3283 \mathrm{~cm}^{-1}$ (C-H stretching), $1630 \mathrm{~cm}^{-1}$ (C=O stretching) and $1541 \mathrm{~cm}^{-1}$ (N-H in-plane bending and C-N stretching) were almost missing after the deproteinization. Immiscible fatty acids such as stearic acid are known to act as nucleating agent of NR crystal [17]. Because the deproteinized and Soxhlet extracted sample did not crystallize even after about one month of storage in the refrigerator due to the absence of fatty acids acting as the nucleating agent, the deproteinized NR sample containing fatty acids was used in this study. (Among fatty acids, miscible ones to NR may depress $T_{m}$ of NR crystal more or less. However, considering the content of the miscible fatty acids, the effect may be limited. The value of $T_{m}^{0}$ estimated in this study could be, therefore, somewhat lower than the real value.)

First, $T_{m}^{0}$ of NR was estimated by the Hoffman-Weeks plot [18]. As a result of the DSC measurements using the conventional DSC instrument along with the XRD-DSC attachment, we obtained the relationship between $T_{c}$ and $T_{m}$. As shown in Fig. 3a, the plot of $T_{m}$ against $T_{c}$ gave a unique straight line, though the data were obtained by two different instruments. The 
intersection point of the extrapolation line of this plot and $T_{m}=T_{c}$ line gives $T_{m}^{0}$. According to Fig. 3a, $T_{m}^{0}$ of NR was estimated to be $61{ }^{\circ} \mathrm{C}$.

$T_{m}^{0}$ of NR was estimated also by the Gibbs-Thomson plot on the basis of Eq. 1. Fig. 4a shows an example of the SAXS intensity profiles after subtraction of the background and air scattering. (In this figure, $q$ is the absolute value of the scattering vector; $q=(4 \pi \sin \theta) / \lambda$ where $\theta$ is the half of the scattering angle.) Crystals of linear NR form the stacked lamellar structure in quiescent state [19]. Therefore, the peak $q$ value in Fig. 4a should correspond to the long period, $L$ of the stacked lamellar structure. The center of the peak was determined by fitting the Gaussian function to the intensity profile (broken line in Fig. 4a). Next the intensity profile was converted to the electron density correlation function $K(z)$ (Fig. $4 \mathrm{~b}, z$ is the length along the stack normal) by the Fourier transformation [16]. In Fig. 4b, $d$ corresponds to the mean thickness of the thinner layer among the crystalline and amorphous layers; the mean thickness of the other (thicker) layer is $(L-d)^{-1}$. Here, we must consider the fact that there is no basis to assume crystalline parts to be uniformly distributed in the specimen. If spherulites are sporadically formed in the amorphous matrix, crystallinity does not correspond to the relative thickness of crystalline layer to the long period. Though crystallinity of NR samples estimated by melting enthalpy was below $50 \%$, the thinner layer can be either the crystalline or the amorphous layer. Therefore, in the Gibbs-Thomson plot (Fig. 4b), $T_{m}$ values obtained by the XRD-DSC attachment was plotted against both $d^{-1}$ and $(L-d)^{-1}$. As shown in Fig. 3b, the plot against $d^{-1}$ crossed the vertical axis $\left(d^{-1}=0\right)$ at $T_{m}=61{ }^{\circ} \mathrm{C}$ (which corresponds to $\left.T_{m}^{0}\right)$, while the plot against $(L-d)^{-1}$ crossed the vertical axis at $T_{m}=48{ }^{\circ} \mathrm{C}$. Considering the result of the Hoffman-Weeks plot, the Gibbs-Thomson plot suggests that the thinner layer should be the crystalline one and $T_{m}^{0}=61^{\circ} \mathrm{C}$.

The value of $T_{m}^{0}$ obtained in this study is higher than all the observed $T_{m} \mathrm{~s}$ in previous studies $[11,13,14]$, and accordingly, far more reasonable than the previously reported value. 
According to Eq. 1, we can also obtain the value of $\sigma_{e}$. Using $\Delta H=6.4 \times 10^{7} \mathrm{Jm}^{-3}$ reported by Kim and Mandelkern [20] and $T_{m}^{0}=334 \mathrm{~K}\left(61{ }^{\circ} \mathrm{C}\right), \sigma_{e}$ was estimated from the slope of the $T_{m}$ vs. $d^{-1}$ plot (Fig. $4 \mathrm{~b}$ ) to be $0.046 \mathrm{Jm}^{-2}$. This value is close to twice the previously reported value of $0.024 \mathrm{Jm}^{-2}[5,6]$.

In this way, previously reported values of $T_{m}^{0}$ and $\sigma_{e}$ were found to be quite different from the ones that were obtained in this study. When strain-induced crystallization of NR is considered on the basis of thermodynamics of polymer crystals, impact of these parameters are so great. We suspect that the absence, even now, of successful thermodynamic theory on straininduced crystallization is, partly, due to use of the inappropriate values of thermodynamic parameters.

\section{Conclusion}

By the Hoffman-Weeks plot and the Gibbs-Thomson plot using lamellar thickness obtained from the same sample as the one used for the DSC measurement, $T_{m}^{0}$ of NR was estimated to be $61{ }^{\circ} \mathrm{C}$. At the same time, $\sigma_{e}$ was estimated to be $0.046 \mathrm{Jm}^{-2}$. Considering that the previously assumed $T_{m}^{0}$ values were inconsistent with experimentally observed $T_{m}$ in some studies [11, $13,14]$, the values estimated in this study should be more reliable.

\section{Acknowledgement}

This work was supported by Grant-in-Aid for Scientific Research (C) (16K05913) from Japan Society for the Promotion of Science (JSPS).

\section{References}

[1] Roberts DE, Mandelkern L. Thermodynamics of Crystallization in High Polymers : Natural Rubbers, J Am Chem Soc. 1955;77;781-786. 
[2] Tanaka Y. Structural characterization of natural polyisoprenes: solve the mystery of natural rubber based on structural study, Rubber Chem Technol. 2001;74;355-375.

[3] Keller A. A Note on Single Crystals in Polymers -Evidence for a Folded Chain Configuration, Phil Mag. 1957;2;1171-1175.

[4] Krigbaum WR, Dawkins JV, Via GH, Belta YI. Effect of strain on the thermodynamic melting temperature of polymers, J Polym Sci., Part A-2. 1966;4;475-489.

[5] Dalal EN, Taylor KD, Philips PJ. The equilibrium melting temperature of cispolyisoprene, Polymer. 1983;24;1623-1630.

[6] Kawahara S, Takano K, Yunyongwattanakorn J, Isono Y, Hikosaka M, Sakdapipanich JT, Tanaka Y. Crystal nucleation and growth of natural rubber purified by deprotenization and trans-esterification, Polymer J. 2004;36;361-367.

[7] Armistead K, Goldbeck-Wood G, Keller A. Polymer Crystallization Theories, Adv Polym Sci. 1992;100;221-312.

[8] Mandelkern L. The role of elastomers in the study of polymer crystallization, Rubber Chem Technol. 1993;66;G61-G75.

[9] Trabelsi S, Albouy P, Rault J. Crystallization and Melting Processes in Vulcanized Stretched Natural Rubber, Macromolecules. 2003;36;7624-7639.

[10] Miyamoto Y, Yamao H, Sekimoto K. Crystallization and Melting of Polyisoprene Rubber under Uniaxial Deformation, Macromolecules. 2003;36;6462-6471.

[11] Valladares D, Yalcin B, Cakmak M. Long Time Evolution of Structural Hierarchy in Uniaxially Stretched and Retracted Cross-Linked Natural Rubber, Macromolecules. 2005;38;9229-9242.

[12] Candau N, Laghmach R, Laurent C, Chenal J, Gauthier C, Biben T, Munch E. StrainInduced Crystallization of Natural Rubber and Cross-Link Densities Heterogeneities, Macromolecules. 2014;47;5815-5824. 
[13] Katzenberg F, Heuwers B, Tiller JC. Superheated Rubber for Cold Storage, Adv Mater. 2011;23;1909-1911.

[14] Tosaka M, Shigeki E. Triaxially Oriented Shape Memory Natural Rubber, Polymer. 2018;157;151-155.

[15] Klinklai W, Saito T, Kawahara S, Tashiro K, Suzuki Y, Sakdapipanich JT, Isono Y. Hyperdeproteinized Natural Rubber Prepared with Urea, J Appl Polym Sci. 2004;93;555-559.

[16] Stroble G, The Physics of Polymers. Berlin Heidelberg: Springer; 1996.

[17] Kawahara S, Kakubo T, Sakdapipanich JT, Isono Y, Tanaka Y. Characterization of fatty acids linked to natural rubber—role of linked fatty acids on crystallization of the rubber, Polymer. 2000;41;7483-7488.

[18] Hoffman JD, Weeks JJ. Melting process and the equilibrium melting temperature of polychlorotrifluoroethylene, J Res Nat Bur Stand. 1962;66A;13-28.

[19] Luch D, Yeh GY. Morpology of strain-induced crystallization of natural rubber.I. Electron microscopy on uncrosslinked thin film, J Appl Phys. 1972;43;4326-4338.

[20] Kim H, Mandelkern L. Multiple Melting Transition in Natural Rubber, J Polym Sci A2. $1972 ; 10 ; 1125-1133$. 


\section{Figure legends}

Fig. 1. Outline of the DSC measurements. (a) Temperature program for crystallization and DSC heating scan. (b) Example of the first and second heating scan. In the first scan, temperature rise is stopped before the complete melting of the seed crystals. Before the second scan, the sample was crystallized at $2.4{ }^{\circ} \mathrm{C}$ for $1 \mathrm{~h}$.

Fig. 2. FT-IR spectrum of untreated and deproteinized NR.

Fig. 3. Estimation of $T_{m}^{0}$. (a) Hoffman-Weeks plot. (b) Gibbs-Thomson plot.

Fig. 4. Extraction of morphological parameters from the SAXS data. (a) Intensity profile. (b) Fourier transform of the intensity profile. The sample was crystallized at $-10{ }^{\circ} \mathrm{C}$ for $2.5 \mathrm{~h}$. 
Fig.1

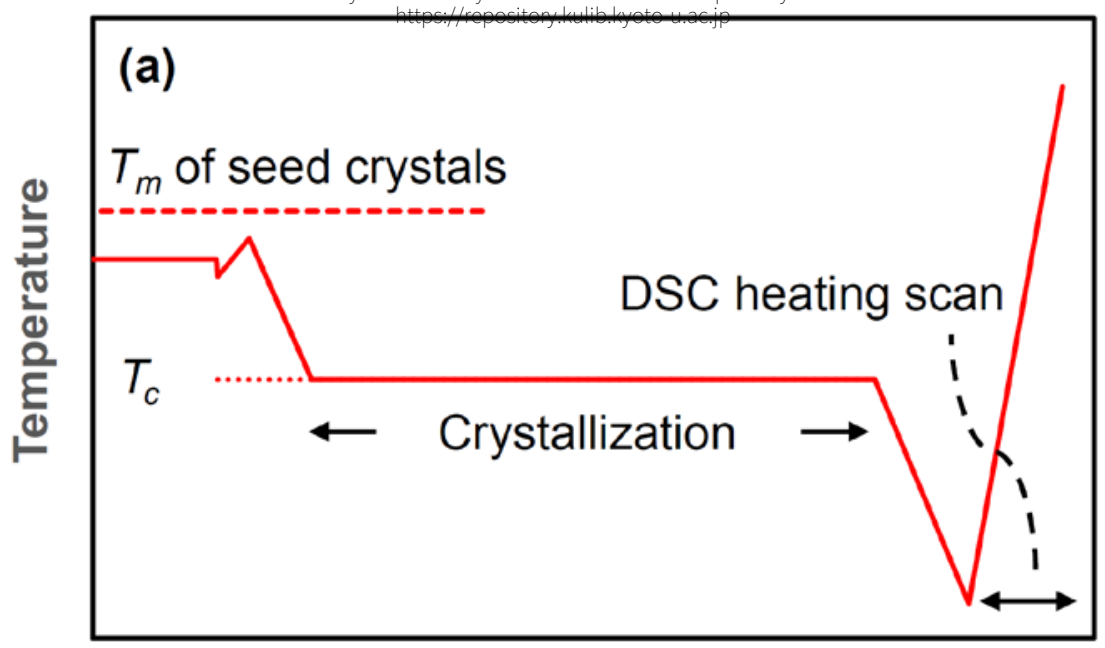

\section{Time}

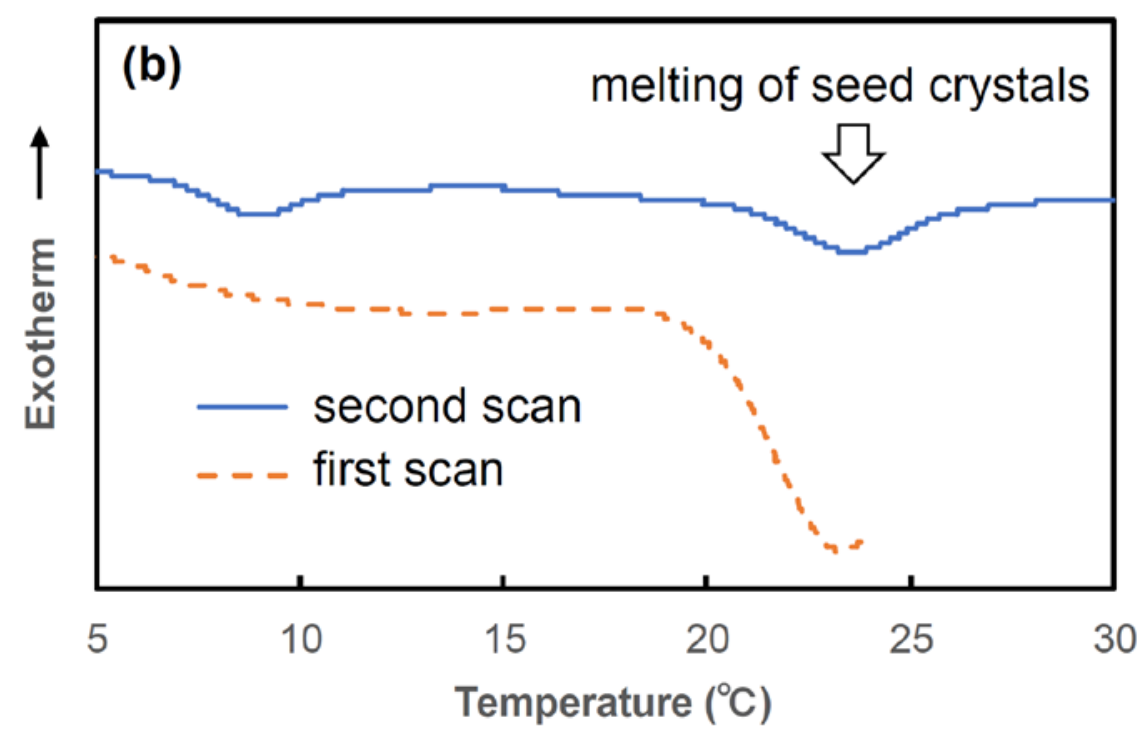

Fig.2

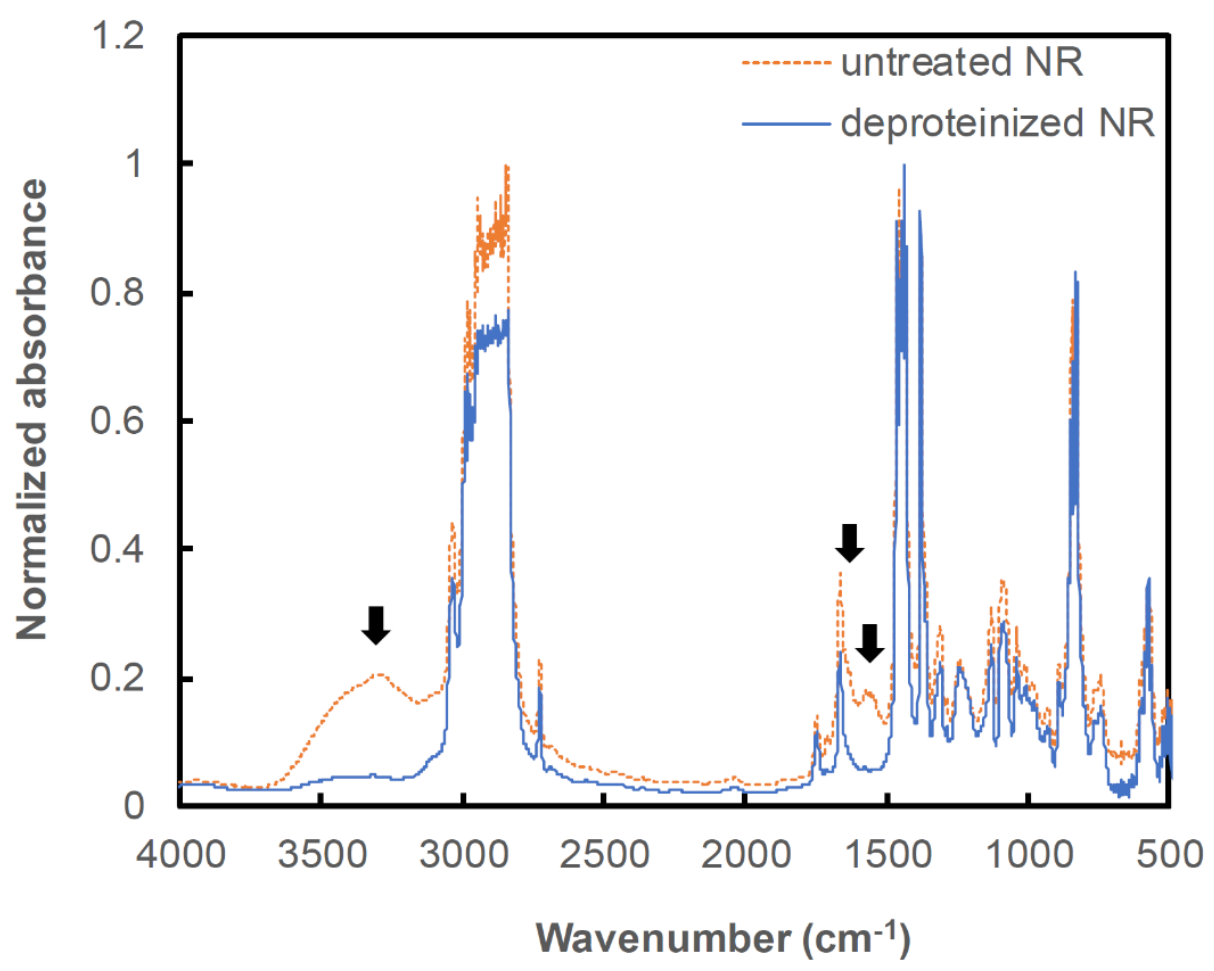


Fig. 3
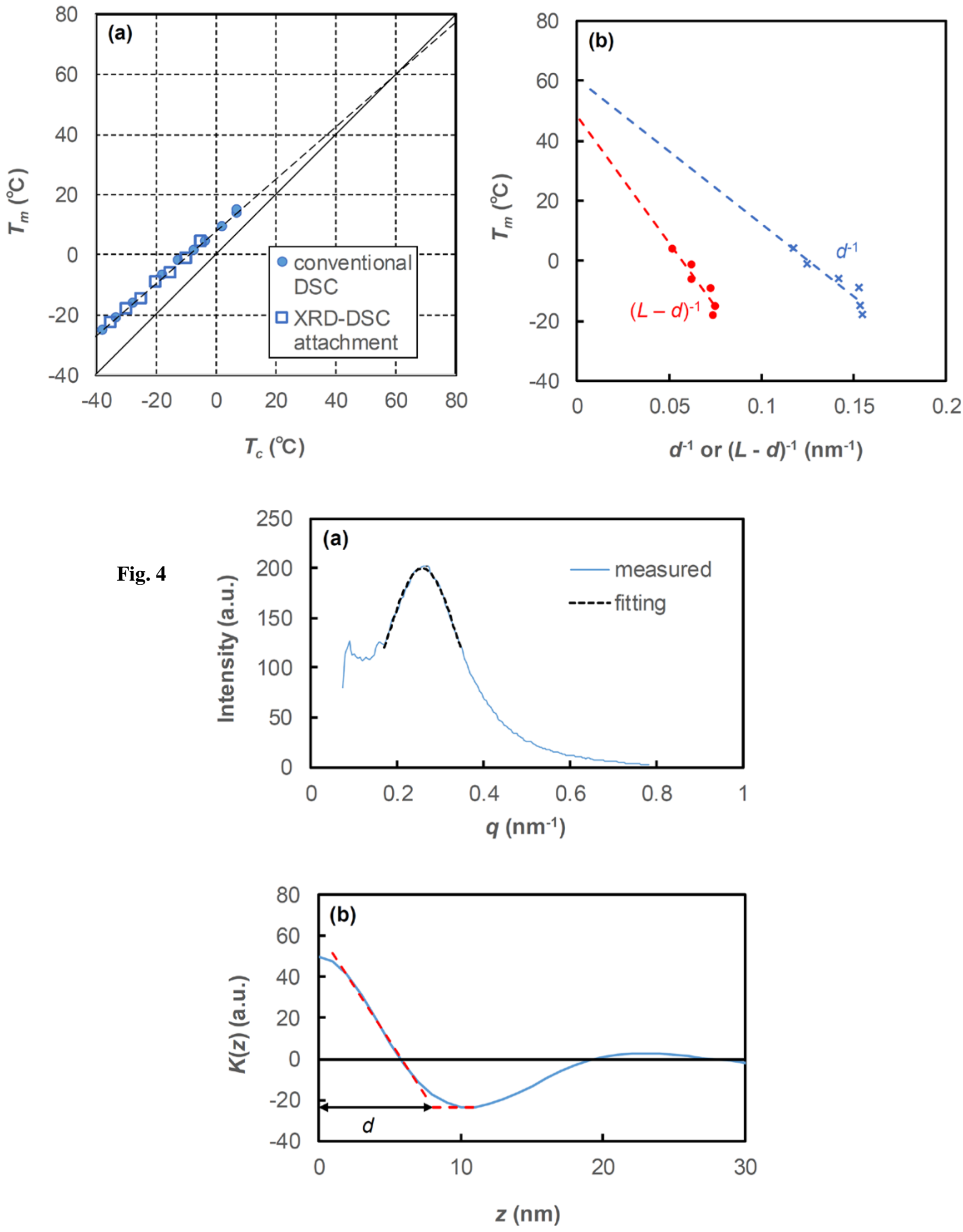